\title{
Molecular characterization of Cyclophilin (TcCyP19) in Trypanosoma cruzi populations susceptible and resistant to benznidazole
}

Juciane Vaz Rêgo ${ }^{\mathrm{a}, \mathrm{b}, \mathrm{e}}$, Ana Paula Duarte ${ }^{\mathrm{b}}$, Daniel Barbosa Liarte $^{\mathrm{c}}$, Francirlene de Carvalho Sousa ${ }^{\mathrm{a}}$, Humberto Medeiros Barreto ${ }^{\mathrm{c}}$, Jacqueline Búa ${ }^{\mathrm{d}}$, Alvaro José Romanha $^{\mathrm{b}}$, Gandhi Rádis-Baptista ${ }^{\mathrm{e}}$, Silvane Maria Fonseca Murta $^{\mathrm{b} *}$

${ }^{a}$ Campus Amilcar Ferreira Sobral, Universidade Federal do Piauí-UFPI, Floriano, PI, Brazil

${ }^{\mathrm{b}}$ Laboratório de Parasitologia Celular e Molecular, Centro de Pesquisa René RachouCPqRR/FIOCRUZ, Belo Horizonte-MG, Brazil

${ }^{\mathrm{c}}$ Campus Petrônio Portela, Universidade Federal do Piauí-UFPI,Teresina-PI, Brazil

dInstituto Nacional de Parasitología “Dr. M. Fatala Chabén”, A.N.L.I.S. Buenos Aires, Argentina

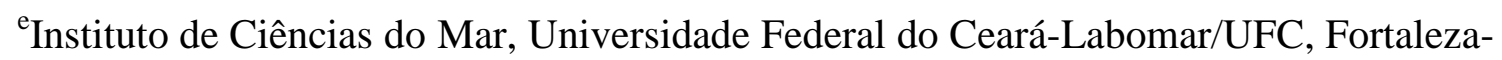
CE, Brazil

* Corresponding author. Tel.: +55-31-33497780, Fax: +55-31-32953115.

E-mail address: silvane@cpqrr.fiocruz.br (S..M.F. Murta), juciane@ufpi.edu.br (J.V. Rego)

Abbreviations: CyP-A, cyclophilin-A; TcCyp19, T. cruzi 19 kDa cyclophilin; BZ, Benznidazole; HGPRT, Hypoxanthine-guanine phosphoribosyltransferase; LIT, Liverinfusion tryptose; NFX, Nifurtimox; PCR, Polymerase chain reaction; qRT-PCR, real time PCR; PFGE, Pulsed-field gel electrophoresis. 


\section{ABSTRACT}

Cyclophilin (TcCyP19), a peptidyl-prolyl cis/trans isomerase, is a key molecule with diverse biological functions that include roles in molecular chaperoning, stress response, immune modulation, and signal transduction. In this respect, TcCyP19 could serve as a potential drug target in disease-causing parasites. Previous studies employing proteomics techniques have shown that the TcCyP19 isoform was more abundant in a benznidazole (BZ)-resistant Trypanosoma cruzi population than in its susceptible counterpart. In this study, TcCyP19 has been characterized in BZ-susceptible and BZresistant $T$. cruzi populations. Phylogenetic analysis revealed a clear dichotomy between Cyphophilin A (CyPA) sequences from trypanosomatids and mammals. Sequencing analysis revealed that the amino acid sequences of TcCyP19 were identical among the T. cruzi samples analyzed. Southern blot analysis showed that $T c C y P 19$ is a single-copy gene, located in chromosomal bands varying in size from 0.68 to $2.2 \mathrm{Mb}$, depending on the strain of T. cruzi. Northern blot and qPCR indicated that the levels of TcCyP19 mRNA were two-fold higher in drug-resistant $T$. cruzi populations than in their drugsusceptible counterparts. Similarly, as determined by two-dimensional gel electrophoresis immunoblot, the expression of TcCyP19 protein was increased to the same degree in BZ-resistant T. cruzi populations. No differences in TcCyP19 mRNA and protein expression levels were observed between the susceptible and the naturally resistant $T$. cruzi strains analyzed. Taken together, these data indicate that cyclophilin TcCyP19 expression is up-regulated at both transcriptional and translational levels in $T$. cruzi populations that were in vitro-induced and in vivo-selected for resistance to BZ.

Key words: Trypanosoma cruzi - Cyclophilin - benznidazole - Drug resistance 


\section{Introduction}

Chagas disease, also known as American trypanosomiasis, is caused by the protozoan parasite Trypanosoma cruzi. It is estimated that 8 to 11 million people are infected with Chagas disease worldwide, mostly in Latin America, where it is endemic (CDC 2013). Drug treatment of chagasic patients currently involves the 5-nitrofuran nifurtimox (NFX) and the 2-nitroimidazole Benznidazole (BZ). However, both drugs cause severe side effects. NFX exerts its action via the generation of nitro anion radicals, a process known as the redox cycle (Maya et al., 2003), while BZ acts via reductive stress, which involves the covalent modification of macromolecules such as DNA, proteins, and lipids by reduced nitro intermediates (Docampo, 1990).

A previous study suggests that both BZ and NFX are prodrugs, activated by nitroreductases to produce nitrogenated radicals (Wilkinson et al., 2008). Interestingly, the deletion of copies of genes encoding two different nitroreductases, namely, old yellow enzyme (TcOYE; also named prostaglandin synthase or NADPH oxidoreductase) (Murta et al., 2006) and trypanosomal type I nitroreductase (NTR-1) (Wilkinson et al., 2008), has been associated with a phenotype of $T$. cruzi, which is resistant to NFX and BZ in vitro. Overexpression of enzymatic activity related to antioxidant response, like that of tryparedoxin peroxidase, ascorbate peroxidase, and iron-superoxide dismutase, has been detected in T. cruzi populations resistant to BZ (Nogueira et al., 2006, 2009, 2012). In addition, the hexose transporter activity was $40 \%$ lower in a BZ-resistant $T$. cruzi population than in the susceptible control (dos Santos et al., 2012). Differences in susceptibility to BZ and NFX between T. cruzi strains (Filardi and Brener, 1987; Murta et al., 1998; Toledo et al., 2004) and/or the genetic diversity of the host (Filardi and Brener, 1987) might explain, in part, the variations in the efficacies 
of antiparasitic drugs. The mechanisms underlying drug resistance in $T$. cruzi and most parasites remain poorly understood. A more in-depth understanding of these mechanisms is essential to develop efficacious chemotherapeutic strategies for fighting Chagas disease.

Several groups have used proteomics approaches in order to understand the mechanisms of drug resistance in protozoan parasites. In a previous study by our group, two-dimensional electrophoresis in combination with mass spectrometry was applied to detect differences in protein expression between BZ-susceptible and -resistant T. cruzi populations, as well as variant clones (Andrade et al., 2008). Among the proteins upregulated in BZ-resistant samples, we observed that cyclophilin TcCyP19 was more abundant in the BZ-resistant population (17LER) and clone (27R) than in their BZsusceptible counterparts (17WTS and clone 9S).

Cyclophilin (TcCyP19) is considered an important player in several biological processes like signal transduction, protein-protein interaction, as well as protein folding and cellular stress response (Galat, 2003). This protein belongs to the peptidyl-prolyl isomerase family (PPIases), which catalyzes the cis-trans isomerization of prolylpeptide bonds in biochemical pathways of cellular communication. The role of Cyclophilin (CyP) in heat shock response, in which the expression of a certain class of cyclophilins was shown to be upregulated under various stressful conditions, has been reviewed previously (Andreeva et al., 1999). The cyclophilin family comprises several isoforms, including cyclophilin TcCyP19, ranging in size from 19 to $110 \mathrm{kDa}$ (Potenza et al., 2006). The most abundantly expressed cyclophilin in the epimastigote stage of $T$. cruzi is TcCyP19. This isoform is a classical cytosolic cyclophilin, with a theoretical mass of $18.782 \mathrm{Da}(19 \mathrm{kDa})$. Búa and co-workers (2001) observed that TcCyP19 is 
homologous to cyclophilin from T. brucei (TbCyP19), Leishmania major (LmCyP19), and T. vivax (TvCyP19). It has been reported that TcCyP19 and other cyclophilin members of T. cruzi (e.g., TcCyP22, TcCyP28, and TcCyP40) are also able to bind to the immunosuppressant drug cyclosporine A (CsA), which could serve to explain their role in mediating immunosuppressive responses (Potenza et al., 2006). Despite its multiple roles, the involvement of TcCyP19 in the BZ-resistant phenotype of $T$. cruzi has not yet been demonstrated.

In the present study, the phylogenetic relationship between TcCyP19 and CyPs from other organisms was established, TcCyP19 nucleotide and amino acid sequence polymorphisms were evaluated, the copy number and chromosomal location of $T c C y P 19$ gene was determined, and the expression of $T c C y P 19$ gene was analyzed at the transcriptional and translational levels. We observed a positive correlation between increased expression of TcCyP19 in T. cruzi populations with in vitro-induced and in vivo-selected resistance to BZ.

\section{Materials and methods}

\subsection{Trypanosoma cruzi strains}

Eleven T. cruzi strains and clones were used in this study (Table I). The BZresistant $T$. cruzi population (17 LER) derived from the Tehuantepec cl2 susceptible wild-type strain (17 WTS) (Nirdé et al., 1995) was obtained by in vitro exposure to increasing concentrations of BZ (LAFEPE Pharmaceutical Laboratory of the State of Pernambuco, Vitória de Santo Antão, Brazil). Parasites of the 17 LER population are resistant to $220 \mu \mathrm{M} \mathrm{BZ}$, a concentration that is 23 -fold higher than the $\mathrm{IC}_{50}$ for the 17 WTS control population. The BZ-resistant T. cruzi population (BZR) was derived from 
the susceptible Y strain (BZS) following in vivo selection after 25 successive passages in mice treated with a single high dose $(500 \mathrm{mg} / \mathrm{kg}$ body weight) of BZ (Murta and Romanha, 1998). We also used two clones derived from these populations: one susceptible (clone 9S) and other resistant (clone 27R). The other five $T$. cruzi strains were previously characterized according to their in vivo susceptibility to BZ and NFX (Filardi and Brener, 1987). The VL-10 strain is naturally resistant to both drugs, while CL Brener, Romano, Buriti and Berenice are susceptible (Murta et al., 1998). All eleven strains were classified as T. cruzi group Tc I, Tc II or Tc VI according to the nomenclature for T. cruzi (Zingales et al., 2009). Epimastigotes forms of all T. cruzi strains were grown in LIT medium, washed in PBS and the parasite pellets were used for the preparation of DNA, RNA and protein samples.

\subsection{In silico and phylogenetic analysis of the $\mathrm{TcCyP} 19$ gene}

Similarity searches were carried out for complete TcCyP19 amino acid sequence (GenBank accession no. XM_816485) within five different $T$. cruzi genomes, using the Basic Local Alignment Search Tool (BLAST; National Center for Biotechnology Information). The genomes used for similarity search were CL Brener Esmeraldo_Like, CLBrener Non-Esmeraldo_like, SylvioX10, JRc14 and Marinkellei_B7 (available in TritrypDB - www.tritrypdb.org - version 6.0). The sequences with high identity values to $T c \mathrm{CyP} 19$ were located within the respective genomes and the predicted proteins were identified using the ARTEMIS (version 15.0 - www.sanger.ac.uk) and BLAST. For phylogenetic analysis, the predicted protein sequences corresponding to cyclophilins were aligned using CLUSTAL-W (Larkin et al., 2007), and the phylogenetic tree was constructed using the MEGA software (Molecular Evolutionary Genetics Analysis, 
version 5.2.2 - Tamura et al., 2011) with bootstrap test (1000 replicates). In addition, the amino acid sequence from $T c \mathrm{CyP} 19$ was compared with related cyclophilin sequences from four species of Trypanosoma (T. brucei, T. congolense, T. evansi and T. vivax), five Leishmania (L. donovani, L. infantum, L. major, L. mexicana and $L$. braziliensis), and two mammals (Mus musculus, and Homo sapiens). A phylogenetic tree for this second multialignment was also constructed using the MEGA software.

\subsection{RNA and DNA preparations}

Genomic DNA and total RNA from $T$. cruzi samples were extracted as previously described (Nogueira et al., 2006). In order to prepare the molecular probes used in the Southern and Northern blot assays, a $400 \mathrm{bp}$ segment corresponding to nucleotide 68 to 468 of the TcCyP19 (GenBank accession no. XM_816485) was amplified from T. cruzi Y strain DNA by conventional PCR using the TcCYP19 forward primer 5' GGTGGCCAATCAGCCGGACG $3^{\prime}$, and the TcCYP19 reverse primer 5', TCCATTGCCTTAACGACTTC 3'. For Southern blot assay, about $5 \mu \mathrm{g}$ total DNA of different $T$. cruzi strains were digested with the restriction enzyme $S a c \mathrm{I}$ in standard reaction conditions (Invitrogen, Carlsbad, CA, USA). The Southern and northern blots were carried out using protocol previously described (Murta et al., 2006).

\subsection{DNA sequencing}

The TcCyP19 534 bp ORF from T. cruzi BZ-susceptible and -resistant populations (17WTS, 17LER, BZS, BZR, CL Brener and VL-10) was cloned into the TOPO PCR2.1 vector (Invitrogen) and amplified in E. coli TOP 10 F' competent cells. Minipreparations of plasmid DNA were done using the QIAprep Spin Miniprep kit 
(Qiagen). Aliquots of $500 \mathrm{ng}$ DNA were sequenced using the DYEnamic WET Dye Terminator Kit (GE Healthcare) in a MegaBACE 1000 DNA Analysis System (GE Healthcare), using the following primers: M13 forward 5'-GTAAAACGACGGCCAG3', M13 reverse 5'-CAGGAAACAGCTATGAC-3' and TcCyP19 forward 5'ATGTCGTACAAGCCGCATCA-3' and TcCyP19 reverse 5'AGGCCTCTGGTCAACTTTAA-3'. Reaction consisted of an initial denaturation at $95^{\circ} \mathrm{C}$ followed by 30 cycles of $15 \mathrm{~s} 95^{\circ} \mathrm{C}, 20$ s at $55^{\circ} \mathrm{C}$ and $80 \mathrm{~s}$ at $60^{\circ} \mathrm{C}$. Samples were analyzed on Mega Bace 400 sequencer (Amersham) and the data were analyzed using Phred, Phrap and Consed. Sequence variability between parasites was assessed by sequencing three colonies of each $T$. cruzi population and by sequencing each colony twice with each primer. Sequences selected for analysis were those with Phred $>40$. Nucleotide sequences were translated into the amino acid sequence using Transec. The nucleotide and amino acid sequences were aligned using the ClustalW 2.1 software.

\subsection{Pulsed Field Gel Electrophoresis (PFGE)}

The $T$. cruzi chromosomes were separated by PFGE in a gene navigator TM system (Amersham Pharmacia, Buckinghamshire, UK), as previously described (Murta et al. 2006). After testing different electrophoresis conditions and switch times, the best condition that provided the optimal separation for $T c C y P 19$ gene was: 70 s for $15 \mathrm{~h}, 90$ $\mathrm{s}$ for $24 \mathrm{~h}, 200 \mathrm{~s}$ for $15 \mathrm{~h}$, and $400 \mathrm{~s}$ for $15 \mathrm{~h}$ at $180 \mathrm{~V}$. After electrophoresis, the gels were transferred onto Hybond nylon membranes (Amersham) as described by the manufacturer's instructions. The membranes were hybridized with the ${ }^{32} \mathrm{P}$-labeled TcCyP19 gene probe. 


\subsection{Quantitative real-time $R T-P C R$}

The protocol employed for the preparation of first strand cDNA and the procedure for real-time RT-PCR were as previously described (Nogueira et al., 2006). An ABI Prism 7000 - Sequence Detection System SDS (PE Applied Biosystems, Foster City, CA, USA) was employed in the real-time PCR amplification of first strand cDNA ( $5 \mu 1)$ using the specific primers: 5' TGTCGTACAAGCCGCATCAC 3' (RT CYPRTF forward) and 5' CAATGCTGACGTCGAAGAAGAC 3' (RT CYPRTR 2 reverse) selected from the complete nucleotide sequence of TcCyP19 (GenBank Accession No. XM_816485). The T. cruzi housekeeping gene hypoxanthine-guanine phosphoribosyltransferase (TcHGPRT) was used to normalize the amount of samples (Nogueira et al., 2006). Standard curves were prepared for each experiment using known quantities of TOPO PCR 2.1 plasmids (Invitrogen) containing the TcCyP19 and TcHGPRT genes. PCR products were quantified using Sequence Detection System data analysis software and normalized to the TcHGPRT values for each sample.

\subsection{Two-Dimensional Gel Electrophoresis (2-DE) and Western blotting analysis}

Protein extracts were obtained by direct lysis of parasites in lysis buffer (Matrangolo et al., 2013) in a proportion of $100 \mu \mathrm{l}$ for $3.5 \times 10^{8}$ epimastigote forms. After extraction, protein concentration was quantified by the Bradford method. Protein extracts $(100 \mu \mathrm{g})$ were loaded on $7 \mathrm{~cm}$ non-linear IPG strips pH 3-10 (Bio-Rad) and isoelectric focused using the Protean IEF Cell (Bio-Rad), $50 \mu \mathrm{A} /$ strip at $20^{\circ} \mathrm{C}$. Passive rehydration was performed for $4 \mathrm{~h}$, followed by an active rehydration at $50 \mathrm{~V}$ for $12 \mathrm{~h}$. Isoelectric focusing was increased gradually to $4,000 \mathrm{~V}$ and run for $16,000 \mathrm{~V}$-hour. Subsequently, the second dimension electrophoretic protein separation was performed 
in $12 \%$ SDS-PAGE and blotted onto nitrocellulose membranes. Blots were incubated for $16 \mathrm{~h}$ at $4^{\circ} \mathrm{C}$ with rabbit polyclonal antibody anti-cyclophilin (TcCyP19) of $T$. cruzi $(1: 5,000)$ (kindly provided by Dr. Jacqueline Búa, Universidad de Buenos Aires, Argentina). The blots were washed and then incubated for $1 \mathrm{~h}$ with horseradish peroxidase-conjugated anti-rabbit IgG 1:2,000 (GE Healthcare). The blots were washed, incubated with ECL Plus chemiluminescent substrate (GE Healthcare) and exposed to X-ray film. To confirm equivalent loading, SDS-PAGE containing the same samples were stained with Colloidal Coomassie Blue G-250 (Neuhoff et al., 1988). The gels stained with Coomassie Blue G250 and the imumnoblots were scanned on a GS-800 calibrated densitometer (BioRad) and submitted to comparative analysis.

\section{Results}

\subsection{In silico and phylogenetic analysis of the $\mathrm{TcCyP} 19$ gene}

Similarity searches were carried out between the $T c C y P 19$ sequence (GenBank accession XM_816485) and a local copy of the $T$. cruzi database of five different $T$. cruzi genomes. Altogether, 79 sequences similar to that of $T c C y P 19$ were found in the five $T$. cruzi genomes (13 to 19 sequences were identified from each genome). The predicted protein sequence of the gene sequences identified was analyzed and compared to the NCBI non-redundant database, and 61 were found to share the highest similarity with cyclophilins. Of these, 24 proteins (including $T c C y P 19)$ had molecular weights ranging from 19 to $23 \mathrm{kDa}$, which is similar to the molecular weight of TcCyP19. A phylogenetic tree was constructed using these 24 cyclophilin sequences (ranging from 18.7 to $23.7 \mathrm{kDa}$ ). A cyclophilin having a molecular weight of $35 \mathrm{kDa}$ was used as the outgroup (Fig. 1S - supplementary data). The results showed that these cyclophilins 
were grouped into two divergent branches. One of the branches included cyclophilins having molecular weights of 18.7 kDa (TcCyP19), 21.4-23.7 kDa, and 21.0-21.1 kDa, while the other encompassed cyclophilins having molecular weights of 21.6-21.7 and $19.7-19.8 \mathrm{kDa}$.

In order to compare the similarity of the TcCyP19 amino acid sequence with cyclophilin-A (CyPA) sequences of different organisms, a neighbor-joining phylogenetic tree was constructed (Fig. 1). This phylogenetic tree shows a clear dichotomous divergence between trypanosomatids and mammalian CyPA sequences. The amino acid sequence of $T c C y P 19$ is closely related to sequences from other species of Trypanosoma and Leishmania, sharing identities ranging between $84 \%$ and $90 \%$ and $78 \%$ and $81 \%$, respectively.

\subsection{Sequencing data}

DNA sequencing of the $T c C y P 19$ gene from $T$. cruzi populations that were susceptible (CL Brener, 17WTS, and BZS), naturally resistant (VL-10), and with in vitro-induced (17 LER) and in vivo-selected (BZS) resistance to BZ was performed in order to investigate whether point mutations could be associated with the BZ-resistance phenotype. Multiple-sequence alignment of the $T c C y P 19$ gene nucleotide sequences revealed three nucleotide mutations (positions 150, 261 and 390) that do not lead to amino acid substitutions (Fig. 2S - supplementary data). No association was found between nucleotide mutations and BZ-resistant phenotype. These three nucleotide mutations are strain-specific.

\subsection{Copy number of the TcCyP19 gene}


Southern blot assays were carried out using samples of $T$. cruzi genomic DNA that had been digested with the endonuclease SacI; which has one restriction site within the reference $T c C y P 19$ sequence (GenBank accession no. XM_816485). Hybridization of the blots of SacI-digested DNA with a TcCyP19-specific probe revealed two fragments of 7.8 and $1.5 \mathrm{~Kb}$ in all T. cruzi samples analyzed (Fig. 3S - supplementary data).

The copy number ratio of the $T c C y P 19$ gene per genome for the pair 17WTS/17LER and BZS/BZR was also determined by real-time PCR. Considering that T. cruzi contains 0.33 pg of DNA (Moser et al., 1989) and that TcHGPRT is a singlecopy gene (Allen and Ullman, 1994), TcCyP19 copy numbers were estimated using $200,100,50$, and $25 \mathrm{ng}$ of genomic DNA. It was found that the copy number ratio of the TcCyP19 gene was the same for $17 \mathrm{WTS} / 17 \mathrm{LER}$ and BZS/BZR populations (data not shown), indicating that the copy number of the TcCyP19 gene is not different in the genome of BZ-resistant T. cruzi populations.

\subsection{Chromosomal location of the $\mathrm{TcCyP} 19$ gene}

Chromosomes from $T$. cruzi strains were separated by pulsed-field gel electrophoresis (PFGE) (Fig. 2A). Chromosome hybridization with the TcCyP19specific probe showed that this gene is present in chromosomal bands that range from 680 to $2200 \mathrm{~kb}$ (Fig. 2B). A correlation between the chromosomal location of the TcCyP19 gene and T. cruzi group was observed for the T. cruzi strains analyzed in this study (Table I). However, no correlation between chromosomal location of the TcCyP19 gene and the drug-resistant phenotype was established. 


\subsection{Levels of TcCyP19 mRNA expression}

Levels of TcCyP19 mRNA in the parasite populations were first investigated by northern blot analysis. A 1.4-kb transcript was detected in northern blots of total RNA derived from BZ-susceptible and -resistant T. cruzi strains, following hybridization with a ${ }^{32} \mathrm{P}$-labelled TcCyP19-specific probe (Fig. 3A). Quantitative controls using a ribosomal RNA probe are shown in Fig. 3B. Comparative densitometric analysis revealed that $T c C y P 19$ mRNA levels were at least 2-fold higher in BZ-resistant 17LER and BZR populations than in their susceptible counterparts 17WTS and BZS. We did not observed any comparative difference in the levels of TcCyP19 mRNA among other T. cruzi strains and clones analyzed.

TcCyP19 mRNA levels were complementarily determined by quantitative realtime RT-PCR to confirm the northern blot results. The amount of TcCyP19 cDNA in the samples of $T$. cruzi was normalized by the single-copy housekeeping gene TcHGPRT, used as an internal reference. The results, shown in Fig 3C, indicate that the levels of transcription of the $T c C y P 19$ gene were 2-fold higher in the 17LER and BZR populations compared to that in the 17WTS and BZS populations. No differences in the levels of transcription of the $T c C y P 19$ gene were detected between the other $T$. cruzi BZ-susceptible and -resistant sample pairs, i.e., CL Brener versus VL-10.

\subsection{Levels of TcCyP19 protein expression}

2-DE western blotting analysis showed that the anti-TcCyP19 polyclonal antibody recognized two spots with the expected size of $19 \mathrm{kDa}$ and an isoelectric point $(\mathrm{p} I)$ of 7.0 to 8.5 in the pair 17LER/17WTS and three spots in the BZR/BZS pair (Fig. 4A), which coincides with that for TcCyP19. These spots, with $\mathrm{p} I$ values ranging from 7.0 to 
8.5, correspond to the different isoforms of TcCyP19, probably owing to posttranslational modifications of the protein (Fig. 4). These findings confirm the protein identity and are suggestive of post-translational modifications of TcCyP19. According to the relevant literature, cyclophilin-A of $L$. infantum has one site of acetylation, justifying the occurrence of isoforms with more acidic $\mathrm{p} I$ (Rosenzweig et al., 2008). In fact, the polyclonal antibody anti-TcCyP19 might recognize TcCyP19 and other cyclophilin isoforms of similar molecular weight but different isoelectric points.

Comparative analysis between Coomassie Blue-stained protein profiles of BZS/BZR and 17WTS/17LER T. cruzi populations showed that the protein load was similar between the samples tested (Fig. 4A and 4C). We selected the same region of both the Coomassie Blue-stained gels of 17WTS/17LER and BZS/BZR to perform the densitometric analysis. In this analysis, the protein expression of spots recognized by anti-TcCyP19 antibody was compared between the 17WTS/17LER and BZS/BZR pairs and normalized using the Coomassie Blue-stained protein profile. Densitometric analysis of the spots showed that the levels of protein expression of the two spots were higher in the 17LER BZ-resistant population than in its susceptible counterpart, i.e., 17 WTS. In the BZS/BZR populations, we identified three spots as cyclophilin TcCyP19. One of them had higher expression levels in the BZS population, while the other two were more highly expressed in the BZ-resistant population BZR than in the susceptible population 17WTS (Fig. 4).

In addition, we comparatively assessed the $\mathrm{TcCyP} 19$ protein expression of a $\mathrm{BZ}$ susceptible T. cruzi strain (CL Brener) and that of a T. cruzi strain (VL-10) naturally resistant to $\mathrm{BZ}$, using western blot one-dimensional gel electrophoresis (1D). No 
differences were observed in the expression levels of TcCyP19 protein between both the T. cruzi strains analyzed (data not shown).

\section{Discussion}

Cyclophilin-A is a member of the peptidyl-prolyl cis/trans isomerase class of enzymes and it is a key molecule in diverse biological processes, including molecular chaperoning, protein folding, and protein trafficking (Bell et al., 2006). In the present study, the gene encoding $T c C y P 19$ was characterized from populations and strains of $T$. cruzi that are susceptible or resistant to benznidazole (BZ). The ORF of TcCyP19 (TcCLB.506925.300) is 534 bp in length and encodes a protein of 177 amino acids, with a predicted mass of $19 \mathrm{kDa}$. Phylogenetic analyses of the amino acid sequences of CyPs revealed a clear dichotomy between trypanosomatid and mammalian cyclophilin sequences. CyP19 proteins from Trypanosoma spp. and Leishmania spp. are the closest related groups in this family. In addition, sequence analysis revealed that the amino acid sequences of the TcCyP19 protein are identical among the $T$. cruzi samples analyzed.

Southern blot analysis showed that the $T c C y P 19$ gene is present in the parasite genome as a single copy in BZ-resistant and -susceptible T. cruzi populations. These data are concordant with the results of a study by Potenza et al. (2006), who reported the presence of a single copy for each of the $T c C y P$ genes analyzed in the $T$. cruzi genome (i.e., TcCyP19, TcCyP20, TcCyp22, TcCyP25, TcCyP28, TcCyP34, and TcCyP40). In the present study, the $T c C y P 19$ gene was located in chromosome bands varying in size from 0.68 to $2.2 \mathrm{Mb}$, depending on the strain of $T$. cruzi. In partial agreement, Búa and co-workers (2001) observed that the TcCyP19 gene is located in two chromosomal bands of sizes 2.0 and $2.2 \mathrm{Mb}$ in the $\mathrm{CL}$ Brener T. cruzi clone, whereas other 
cyclophilin isoforms are found dispersed in the T. cruzi genome. These differences could be due to $T$. cruzi chromosomal rearrangements or cyclophilin-like sequences that could be recognized by the $T c C y P 19$ probe.

Northern blot and qPCR indicated that the levels of $T c C y P 19$ mRNA were twofold higher in T. cruzi populations with in vitro-induced and in-vivo selected resistance to $\mathrm{BZ}$ compared to that in drug-susceptible counterparts. In accordance with these results, analytical 2DE immunoblot showed an increase in TcCyP19 protein expression level in the BZ-resistant T. cruzi populations. However, no differences in TcCyP19 mRNA and protein expression levels were observed between the susceptible and naturally resistant $T$. cruzi strains analyzed, indicating different drug resistance mechanisms in these samples. Many studies evaluating drug resistance mechanisms in parasites were based on models produced by artificial induction of resistance. In contrast, there is very little information available on the biochemical mechanisms underlying drug resistance in field isolates. The data presented here show that TcCyP19 protein expression level is increased in $T$. cruzi populations that were in vitro-induced and in-vivo selected for resistance to BZ, a situation that is different from that observed in the naturally resistant population. In agreement with our results, Villareal et al. (2005) observed that the mechanisms associated with natural resistance to drugs differ from those associated with induced resistance. The mechanism of drug resistance, such as that to BZ, is often complex and multifactorial, including molecules associated with the host immune system, which may interfere with the susceptibility of the parasite to the drug (Murta et al., 1999). Studies to determine whether overexpression of TcCyP19 in the susceptible population will confer the BZ-resistant phenotype to these parasites 
are required to confirm our hypothesis that TcCyP19 may be involved in $T$. cruzi resistance to $\mathrm{BZ}$.

Interestingly, in the presence of BZ or other stress conditions, an increase in the expression of several proteins of distinct biological function is observed. Consequently, TcCyP19 might contribute to counteract the chemical stress stimulus by increasing, for example, the activity of protein folding, which is necessary for efficient expression of functional polypeptides. The correlation between drug resistance and increased chaperonin activity has been observed in L. donovani (Kumar et al., 2010) and T. cruzi (Andrade et al., 2008). Interestingly, CyPA also has a protective role in murine cells, functioning as an antioxidant against oxidative stress (Doyle et al., 1999; Hong et al., 2004). Additionally, in humans, CyPA binds peroxiredoxins, thereby increasing the peroxidase activity by their ability to transfer electrons (Lee et al., 2001). In analogy to this role, our data are indicative that the increased expression of TcCyP19 could in turn favor the expression and activity of enzymes associated with antioxidant defense (Nogueira et al., 2006, 2009, 2012). This might serve in detoxifying the parasite and might confer BZ-resistance - a hypothesis that warrants further investigation.

Cyclophilin-A is also implicated in biological processes such as tumor resilience and progression. For instance, microarray analysis has shown that CyPA can upregulate the expression of cytokines and genes related to drug resistance (Chen et al., 2008). Furthermore, it was observed that elevated CyPA expression contributes to the drugresistance phenotype in cancer cells (Yang et al., 2011). Another study revealed that the overexpression of CyPA could promote cancer cell proliferation, cell migration/invasion, apoptosis inhibition, and drug-resistance phenotype in various cancer cell types (Obchoei et al., 2009). These data suggest that CyPA is a good target 
for cancer chemotherapy. Interestingly, some studies suggest that TcCyPs are promising targets for the treatment of Chagas disease (Búa et al., 2008; Carraro et al., 2007). Cyclophilins bind cyclosporin A (CsA), an immunosuppressive antimicrobial drug, and non-immunosuppressive CsA analogues possess higher activity against $T$. cruzi (Búa et al., 2008). In conclusion, given that our results show that TcCyP19 is upregulated in BZ-resistant T. cruzi populations and that several lines of evidence implicate CyPs as a promising chemotherapeutic target against cancer and parasitic diseases, our work constitutes a starting point for further investigation into the role of TcCyPs in the mechanism of drug resistance and as a target for Chagas disease chemotherapy.

Acknowledgements The authors are grateful to the Program for Technological Development in Tools for Health-PDTIS-FIOCRUZ for the use of facilities. Financial support for the study was received from the following Brazilian agencies: Conselho Nacional de Desenvolvimento Científico e Tecnológico (CNPq/Universal 475782/20127), Fundação de Amparo à Pesquisa do Estado de Minas Gerais (FAPEMIG - CBBPPM 00536/11 and CBB - PPM-00196-13) and P3D-Programa de descoberta e desenvolvimento de drogas (PROEP/CNPq/FIOCRUZ 401988/2012-0).

\section{References}

Allen, T., Ullman, B., 1994. Molecular characterization and overexpression of the hypoxantine-guanine phosphoribosyltransferase gene from Trypanosoma cruzi. Mol. Biochem. Parasitol. 65, 233-245.

Andrade, H.M., Murta, S.M., Chapeaurouge, A., Perales, J., Nirdé, P., Romanha, A.J., 2008. Proteomic analysis of Trypanosoma cruzi resistance to benznidazole. J. Proteome Res. 7, 2357-2367.

Andreeva, L., Heads, R, Green, C.J., 1999. Cyclophilins and their possible role in the stress response. Int. J. Exp. Pathol. 80, 305-315. 
Bell, A., Monaghan, P., Page, A.P., 2006. Peptidyl-prolyl cis-trans isomerases (immunophilins) and their roles in parasite biochemistry, host-parasite interaction and antiparasitic drug action. Int. J. Parasitol. 36, 261-276.

Búa, J., Aslund, L., Pereyra, N., Garcia, G.A., Bontempi E.J., Ruiz A.M., 2001. Characterisation of a cyclophilin isoform in Trypanosoma cruzi. FEMS Microbiol. Lett. 200, 43-47.

Búa, J., Fichera, L.E., Fuchs, A.G., Potenza, M., Dubin, M., Wenger, R.O., Moretti, G., Scabone, C.M., Ruiz, A.M., 2008. Anti-Trypanosoma cruzi effects of cyclosporin A derivatives: possible role of a P-glycoprotein and parasite cyclophilins. Parasitol. $135,217-228$.

Carraro, R., Búa, J., Ruiz, A., Paulino, M., 2007. Modelling and study of cyclosporin A and related compounds in complexes with a Trypanosoma cruzi cyclophilin. J Mol Graph Model. 26:48-61.

CDC, 2013. Centers for Disease Control and Prevention, (Last updated July 19, 2013). American trypanosomiasis. Available: 〈http://www.cdc.gov/parasites/chagas/>.

Chen, S., Zhang, M., Ma, H., Saiyin, H., Shen, S., Xi, J., Wan, B., Yu, L., 2008. OligosMicroarray analysis reveals the role of cyclophilin a in drug resistance. Cancer Chemother. Pharmacol. 61, 459-69.

Docampo, R., 1990. Sensitivity of parasites to free radical damage by antiparasitic drugs. Chem. Biol Interact. 73, 1-27.

dos Santos, P.F., Ruiz, J.C., Soares, R.P., Moreira, D.S., Rezende, A.M., Folador, E.L., Oliveira, G., Romanha, A.J., Murta, S.M., 2012. Molecular characterization of the hexose transporter gene in benznidazole resistant and susceptible populations of Trypanosoma cruzi. Parasit. Vectors 5, 161.

Doyle, V., Virji, S., Crompton, M., 1999. Evidence that cyclophilin-A protects cells against oxidative stress. Biochem. J. 341, 127-132.

Efron, B., Halloran, E., Holmes, S., 1996. Bootstrap confidence levels for phylogenetic trees. Proc. Natl. Acad. Sci. U.S.A. 93, 13429-13434

Filardi, L.S., Brener, Z., 1987. Susceptibility and natural resistance of Trypanossoma cruzi strains to drugs used clinically in Chagas Disease. Trans. R. Soc. Trop. Med. Hyg. 81, 755-759.

Galat, A., 2003. Peptidylprolyl cis/trans isomerases (immunophilins): biological diversity--targets--functions. Curr. Top. Med. Chem. 3, 1315-1347.

Hong, F., Lee, J., Piao, Y.J., Jae, Y.K., Kim, Y.J., Oh, C., Seo, J.S., Yun, Y.S., Yang, C.W., Ha, J., Kim, S.S., 2004. Transgenic mice overexpressing cyclophilin A are 
resistant to cyclosporin A-induced nephrotoxicity via peptidyl-prolyl cis-trans isomerase activity. Biochem. Biophys. Res. Commun. 316, 1073-1080.

Kumar, A., Sisodia, B., Misra, P., Sundar, S., Shasany, A.K., Dube, A. 2010. Proteome mapping of overexpressed membrane-enriched and cytosolic proteins in sodium antimony gluconate (SAG) resistant clinical isolate of Leishmania donovani. Br. J. Clin. Pharmacol. 70, 609-617.

Larkin, M.A., Blackshields, G., Brown, N.P., Chenna, R., McGettigan, P.A., McWilliam, H., Valentin, F., Wallace, I.M., Wilm, A., Lopez, R., Thompson, J.D., Gibson, T.J., Higgins, D.G. 2007. Clustal W and Clustal X version 2.0. Bioinformatics. 23, 2947-2948.

Lee, S.P., Hwang, Y.S., Kim, Y.J., Kwon, K.S., Kim, H.J., Kim, K., Chae, H.Z., 2001. Cyclophilin-A binds to peroxiredoxins and activates its peroxidase activity. J. Biol. Chem. 276, 29826-29832.

Matrangolo, F.S., Liarte, D.B., Andrade, L.C., de Melo, M.F., Andrade, J.M., Ferreira, R.F., Santiago, A.S., Pirovani, C.P., Silva-Pereira, R.A., Murta, S.M., 2013. Comparative proteomic analysis of antimony-resistant and -susceptible Leishmania braziliensis and Leishmania infantum chagasi lines. Mol. Biochem. Parasitol. 190, 63-75.

Maya, J.D., Bollo, S., Nunez-Vergara, L.J., Squella, J.A., Repetto, Y., Morello, A., Pèrie, J., Chauviere, G., 2003. Trypanosome cruzi: effect and mode of action of nitroimidazole and nitrofuran derivates. Biochem. Pharmacol. 65, 999-1006.

Moser, D.R., Kirchhoff, L.V., Donelson, J.E., 1989. Detection of Trypanosoma cruzi by DNA amplification using the polymerase chain reaction. J. Clin. Microbiol. 27, 1477-1482.

Murta, S.M., Gazzinelli, R.T., Brener, Z., Romanha, A.J., 1998. Molecular characterization of susceptible and naturally resistant strains of Trypanosoma cruzi to benznidazole and nifurtimox. Mol. Biochem. Parasitol. 93, 203-214.

Murta, S.M., Krieger, M.A., Montenegro, L.R., Campos, F.F., Probst, C.M., Avila, A.R., Muto, N.H., De Oliveira, R.C., Nunes, L.R., Nirdé, P., Bruna-Romero, O., Goldenberg, S., Romanha, A.J., 2006. Deletion of copies of the gene encoding old yellow enzyme (TcOYE), A NAD $(\mathrm{P}) \mathrm{H}$ flavin oxidoreductase, associates with in vitro-induced benznidazole resistance in Trypanosoma cruzi. Mol. Biochem. Parasitol. 146, 151-162.

Murta, S.M., Romanha, A.J., 1998. In vivo selection of a population of Trypanosoma cruzi and clones resistant to benzinidazole. Parasitol. 116, 165-171.

Murta, S.M.F., Ropert, C., Alves, R.O., Gazzinelli, R.T., Romanha, A.J., 1999. In vivo treatment with benznidazole enhances phagocytosis, parasite destruction and cytokine release by macrophages during infection with a drug-susceptible but not with a derived drug-resistant Trypanosoma cruzi population. Parasite Immunol. 21, 535-544. 
Neuhoff, V., Arold, N., Taube, D., Ehrhardt, W., 1988. Improved staining of proteins in polyacrylamide gels including isoelectric focusing gels with clear background at nanogram sensitivity using Coomassie Brilliant Blue G-250 and R-250. Electrophoresis. 9, 255-262.

Nirdé, P., Larroque, C., Barnabé, C., 1995. Drug resistant epimastigotes of Trypanosoma cruzi and persistence of this phenotype after differentiation into amastigotes. C. R. Acad. Sci. Ser. III 318, 1239-1244.

Nogueira, F.B., Krieger, M.A., Nirdé, P., Goldenberg, S., Romanha, A.J., Murta, S.M., 2006. Increased Expression of Iron-containing superoxide dismutase-A (TcFESODA) enzyme in Trypanosoma cruzi population within vitro-induced resistance to benznidazole. Acta Trop. 100, 119-132.

Nogueira, F.B., Rodrigues, J.F., Correa, M.M., Ruiz, J.C., Romanha, A.J., Murta, S.M., 2012. The level of ascorbate peroxidase is enhanced in benznidazole-resistant populations of Trypanosoma cruzi and its expression is modulated by stress generated by hydrogen peroxide. Mem. Inst. Oswaldo Cruz 107, 494-502.

Nogueira, F.B., Ruiz, J.C., Romanha, A.J., Murta, S.M.F., 2009. Molecular characterization of cytosolic and mitochondrial tryparedoxin peroxidase in Trypanosoma cruzi populations susceptible and resistant to benznidazole. Parasitol. Res. 104, 835-844.

Obchoei, S., Wongkhan, S., Wongkham, C., Li, M., Yao, Q., Chen, C., 2009. Cyclophilin A: potential functions and therapeutic target for human cancer. Med. Sci. Monit. 5, 221-232. Review.

Potenza, M., Galat, A., Mining, T.A., Ruiz, A.M., Duran, R., Tarleton, R.L., Marin, M., Fichera, L.E., Búa, J., 2006. Analysis of the Trypanosoma cruzi cyclophilin gene family and identification of cyclosporin a binding proteins. Parasitol. 132, 867-882.

Rosenzweig, D., Smith, D., Myler, P.J., Olafson, R.W., Zilberstein, D., 2008. Posttranslational modification of cellular proteins during Leishmania donovani differentiation. Proteomics. 8, 1843-1850.

Tamura, K., Peterson, D., Peterson, N., Stecher, G., Nei, M., Kumar, S., 2011. MEGA5: molecular evolutionary genetics analysis using maximum likelihood, evolutionary distance, and maximum parsimony methods. Mol. Biol. Evol. 28, 2731-2739.

Toledo, M.J., Bahia, M.T., Veloso, V.M., Carneiro, C.M., Machado-Coelho, G.L., Alves, C.F., Martins, H.R., Cruz, R.E., Tafuri, W.L., Lana, M., 2004. Effects of specific treatment on parasitological and histopathological parameters in mice infected with different Trypanosoma cruzi clonal genotypes. J. Antimicrob. Chemother. 53, 1045-1053. 
Villarreal, D., Nirde, P., Hide, M., Barnabe, C., Tibayrenc, M., 2005. Differential gene expression in benznidazole-resistant Trypanosoma cruzi parasites. Antimicrob. Agents Chemother. 49, 2701-2709.

Wilkinson, S.R., Taylor, M.C., Horn, D., Kelly, J.M., Cheeseman, I., 2008. A mechanism for cross-resistance to nifurtimox and benznidazole in trypanosomes. Proc. Natl. Acad. Sci. U.S.A. 105, 5022-5027.

Yang, J., Li, A, Yang, Y., Li, X., 2011. Identification of cyclophilin A as a potential prognostic factor for clear-cell renal cell carcinoma by comparative proteomic analysis. Cancer Biol. Ther. 11, 535-46.

Zingales, B., Andrade, S.G., Briones, M.R., Campbell, D.A., Chiari, E., Fernandes, O., Guhl, F., Lages-Silva, E., Macedo, A.M., Machado, C.R., Miles, M.A., Romanha, A.J., Sturm, N.R., Tibayrenc, M., Schijman, A.G., 2009. Second Satellite Meeting. A new consensus for Trypanosoma cruzi intraspecific nomenclature: second revision meeting recommends TcI to TcVI. Mem. Inst. Oswaldo Cruz 104, 10511054. 


\section{Legends to Figures - Rego et al.}

Fig. 1: Neighbour-joining phylogenetic tree of CyPs sequences of Trypanosoma cruzi and other organisms with a high similarity degree. The numbers shown are bootstrap values. Bootstrap is a method that provides assessments of confidence for each clade of an observed tree, based on the proportion of bootstrap trees showing that same clade (Efron et al., 1996).

Fig. 2: Chromosomal location of the $T c C y P 19$ gene in BZ-resistant and susceptible $T$. cruzi strains. (A) Chromosomal bands from the T. cruzi strains were separated by PFGE and stained with ethidium bromide. (B) Southern blots of the chromosomes were hybridised with a ${ }^{32} \mathrm{P}$-labeled $T c C y P 19$-specific probe. Whole chromosomes from Saccharomyces cerevisae were used as molecular weight markers

Fig. 3: Levels of $T c C y P 19$ mRNA in BZ-resistant and susceptible T. cruzi strains. (A) Northern blot profile of total RNA extracts from the T. cruzi strains obtained using a ${ }^{32} \mathrm{P}$-labeled TcCyP19 -specific probe. (B) The quantitative control was used agarose gel containing the RNA, stained with ethidium bromide. (C) Number of cDNA molecules (copy number ratio) of $T c C y P 19\left(\mathrm{x} 10^{6}\right)$. Values were normalized to those obtained for the TcHGPRT and are presented as the means ( \pm S.D.M.) of triplicate real-time RT-PCR analyses from three independent experiments.

Fig. 4: 2-DE gels of proteins and Western blot analysis of TcCyP19 expression in the benznidazole-susceptible and -resistant T. cruzi populations. Proteins $(100 \mu \mathrm{g})$ were loaded on $7 \mathrm{~cm}$, non-linear IPG strips of $\mathrm{pH} 3-10$, submitted to isoelectric focusing and separated on $12 \%$ SDS-PAGE. The gels were stained with Colloidal Coomassie Blue G250 (A and C) or blotted onto nitrocellulose membranes (B and D). The blots were probed with a rabbit polyclonal antibody anti-cyclophilin-A of $T$. cruzi $(1: 5,000)$ and developed using ECL. 


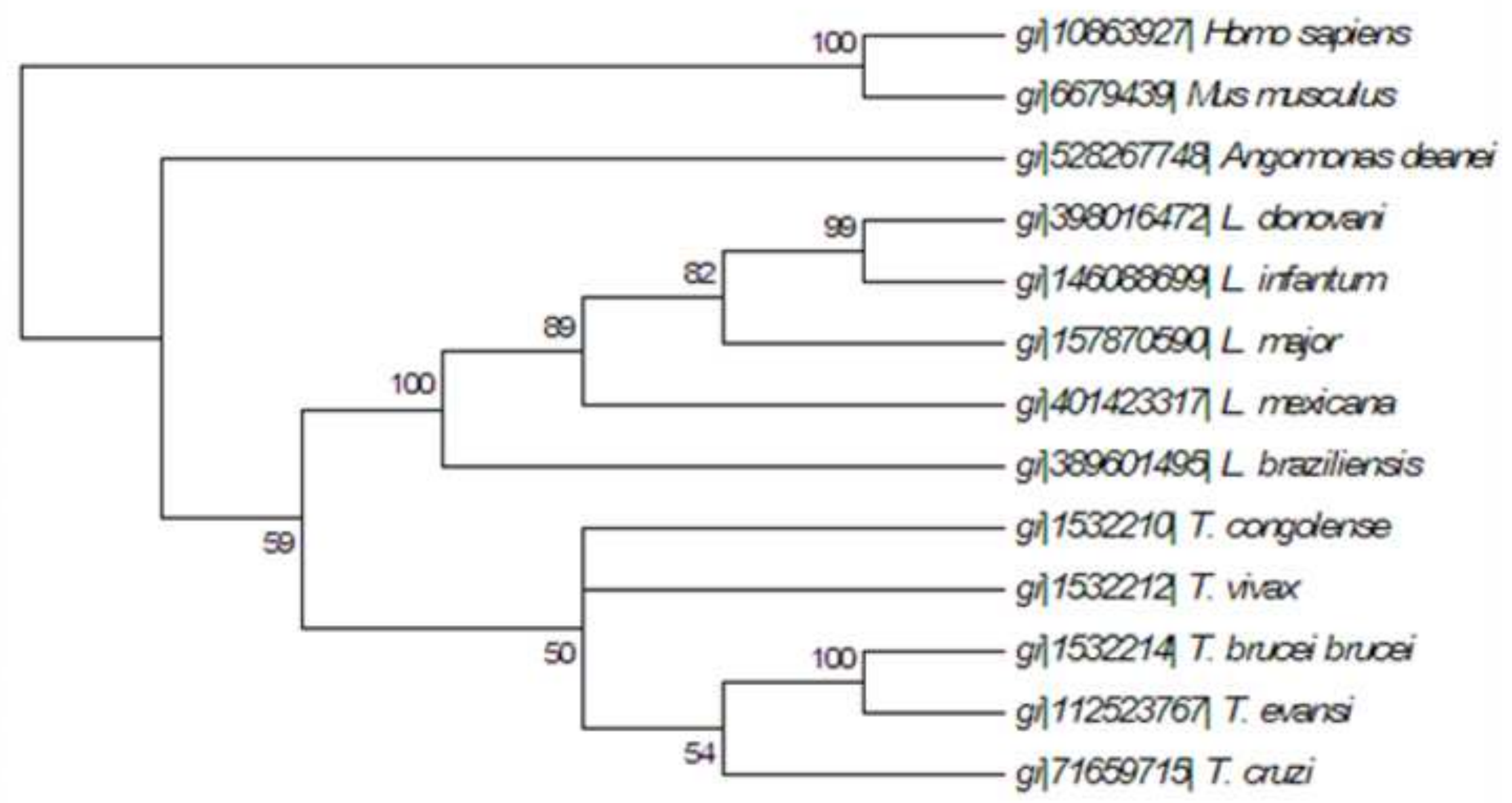


Click here to download high resolution image
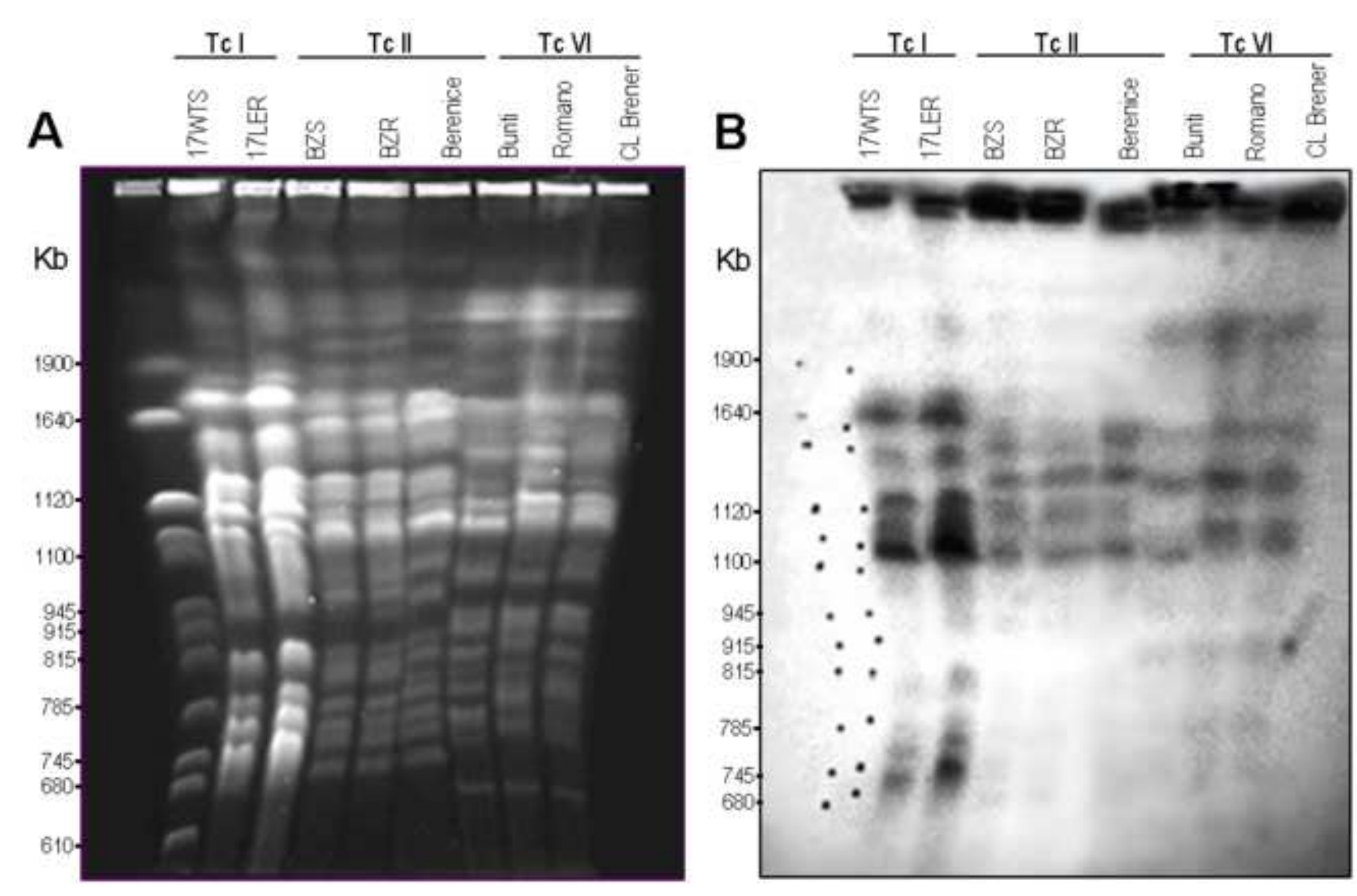

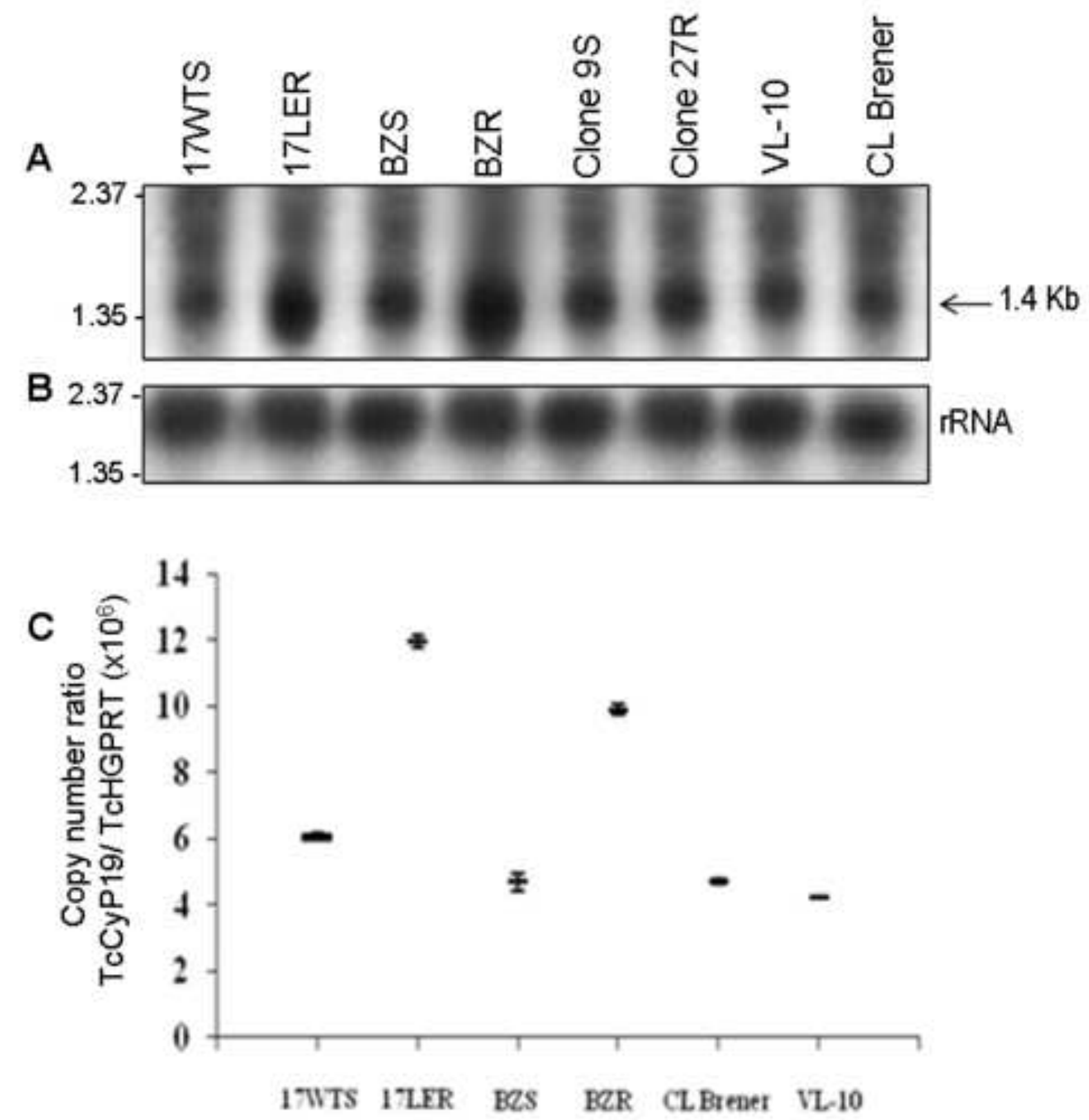
Click here to download high resolution image

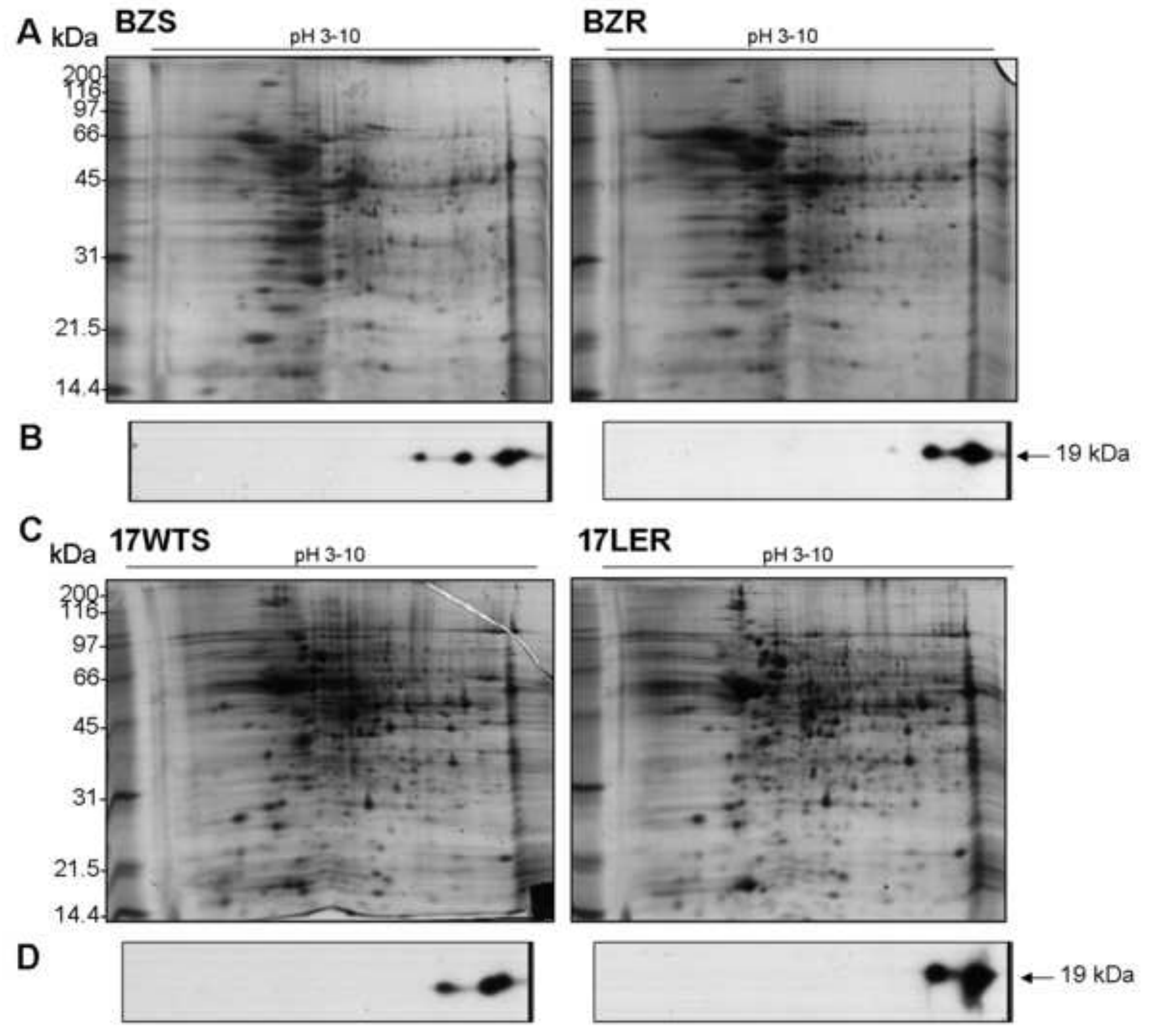

